REVISTA DE DERECHO UNED, núm. 1, 2006

\title{
LA CONVERGENCIA CURRICULAR EN LOS ESTUDIOS DE LAS CIENCIAS SOCIALES
}

ALEJANDRo CONDE LÓPEZ

Sumario: UNA INTRODUCCIÓN A LOS PRINCIPIOS DE LA CONVERGENCIA CURRICULAR.-CRITERIOS EN EL DISEÑO E IMPLANTACIÓN DE UN PROCESO DE FLEXIBILIZACIÓN CURRICULAR.-LA DESCONEXIÓN SECULAR ENTRE LA UNIVERSIDAD Y LA EMPRESA.-UN MOMENTO PARA EL CAMBIO.-EL PROYECTO DE MODELO DEL ESPACIO EUROPEO DE LA EDUCACIÓN SUPERIOR.-LAS DIFICULTADES EN LA APLICACIÓN DEL PROYECTO.-LA IMPLANTACIÓN DEL MODELO EUROPEO EN ESPAÑA.-LA PROYECCIÓN EN IBEROAMÉRICA DE LA CONVERGENCIA CURRICULAR Y CONCLUSIONES.-BIBLIOGRAFÍA.

El siglo XXI ha comenzado, empujado por los acontecimientos de finales del anterior, lleno de cambios y de retos. El desmoronamiento de las rígidas estructuras socialistas en los países del este de Europa y el rotundo triunfo del no tan perfecto sistema capitalista nos ha llevado, en todo género de cosas, a la denominada "Globalización». Junto a ella, un sinfín de adelantos informáticos y en las comunicaciones que han trastocado, mejor dicho, que están trastocando, toda la estructura científico social de la humanidad.

La crisis de la última década del siglo $\mathrm{xx}$ ha vuelto a plantear nuevas exigencias al sistema educativo existente. La preocupación fundamental va a centrarse en el empleo y la capacitación de los universitarios ante el doble reto de estar mejor preparados para el trabajo productivo y de ser capaces de adaptarse a los cambios tecnológicos y estructurales que se están produciendo en la sociedad y, 
sobre todo, la competencia de otros países más preparados técnica y culturalmente.

El mundo actual es el mundo de la competencia. Es un desafío continuo, sin precedentes y donde todo y todos nos comparamos para poder elegir lo mejor y al mejor. Criticamos y tememos a los chinos porque, gracias a este fenómeno de liberalismo, globalización y comunicación, producen bien y a precios mucho más bajos que el resto de los países. Los científicos, los economistas, los sociólogos, temen ese futuro de la competencia y desconocen lo que va a ocurrir. Ante este desconcierto es, pues, necesario la reflexión. Es en este confuso entorno donde, en todos los niveles, se hace imperiosa la necesidad de planes de acción. Junto y frente al fenómeno de la globalización y de la internacionalización, se hace ineludible la apertura de fronteras, tanto técnicas como económicas y el intercambio, no solo de mercancías, sino de tecnología y, sobre todo, de conocimientos.

\section{UNA INTRODUCCIÓN A LOS PRINCIPIOS DE LA CONVERGENCIA CURRICULAR}

Se precisa de la homologación y con ella de la internacionalización del ejercicio profesional y, para ello, se hace igualmente necesaria la convergencia curricular, tanto en los estudios primarios como de grado y postgrado, incluso en los de enseñanza permanente. En definitiva, hemos de alcanzar niveles óptimos de estandarización que nos haga no sólo competitivos, sino «normalizados», homologados, comparables e intercambiables con los demás. Esta amplia problemática, evidentemente, abarca mucho más allá de la enseñanza que es el epicentro al que en este trabajo nos referimos. Lo que en otras áreas es de evidente necesidad, aquí en los estudios superiores, se hace, sin embargo, imprescindible.

La enseñanza ha estado desde hace mucho tiempo en la mayoría de los países europeos y especialmente en España, con una casi total desconexión con el mundo real, profesional, de la economía y de la empresa. Los planes de estudio de las distintas carreras universitarias, sobre todo las jurídicas, tenían un corte generalista y presentaban una especialización prácticamente nula.

Continuamente hay que preguntarse el por qué un abogado norteamericano, británico o alemán tienen una profesión tan bien considerada como retribuida, mientras que su homónimo español, por ejem- 
plo, salvo excepciones, sufría la humillación de conformarse con cualquier puesto, incluso, como se decía, de tranviario...Si hubiéramos comparado los estudios en estos países y la seriedad de los planes de los mismos, nos daríamos cuenta de las razones de estas diferencias.

En este trabajo, aunque sea imposible profundizar a causa de su escueta dimensión, vamos a pretender convencer de la necesidad de llevar a cabo una formación curricular modular, innovadora y flexible en nuestros estudios universitarios, incluso de postgrado y muy especialmente en estas Facultades, como son las de Ciencias Sociales. (Económicas, Políticas o Derecho) en las que, tradicionalmente, han predominado los estudios tan sesgados en una u otra dirección político-religiosa, como poco prácticos.

En el mundo actual se precisa una Universidad que esté más conectada con el desarrollo científico-tecnológico, social, cultural y práctico porque esto es básico para su posicionamiento en un mundo que día a día es más globalizado y competitivo. Y todo ello, además, como consecuencia de una posición de inferioridad histórica del atraso de nuestras Universidades que se ven ahora obligadas a tener que sufrir la competencia de los técnicos, científicos y en nuestro caso de los juristas, economistas o sociólogos, mucho mejor preparados, de nuestro entorno comunitario de la Unión Europea.

La libertad e igualdad de oportunidades es consecuencia de la educación como derecho que avoca en la educación como mercado y en la educación como tarea moral, arcano desde donde se distribuyen los conocimientos técnicos y científicos. Todo ello va a conformar esas misteriosas fuerzas de cambio a nivel mundial que tanto temen algunos, en vez de enfrentarse a ellas, a ese apasionante reto del que todos nos podremos beneficiar.

Así, centrándonos en nuestro análisis, diremos que nos enfrentamos a un nuevo enfoque profesional que precisa de una urgente modernización de nuestros conocimientos que han de ser mucho más prácticos y operativos que los planes tradicionalmente estudiados, por ejemplo, en nuestras Facultades de Derecho.

Hemos de modificar nuestros paradigmas educacionales, aplicando nuevas tecnologías de enseñanza en un proceso más realista de instrucción - aprendizaje, centrado en el estudiante y no en los intereses de ciertos profesores. Globalización; internacionalización; apertura de fronteras; ejercicio profesional y la imprescindible armonización. Aprender a conocer; aprender a hacer; aprender a vivir juntos y con los demás y también, aprender a emprender como ya 
destacó por la UNESCO en el «Informe de la Comisión Delors» de 1995.

Para hacer frente a estos retos, para conseguir la pretendida y necesaria armonización curricular de los estudios universitarios europeos, las universidades de 30 países de Europa están trabajando en la creación de un espacio universitario europeo. Este espacio común y homogéneo es la meta última del controvertido "Proceso de Bolonia» iniciado en 1999. Este proceso y su programa básico se complementaría, además, con el desarrollo del "VI Programa Marco» cuya intención es crear, además, un Espacio Europeo de Investigación con trascendencia en ciertas universidades iberoamericanas. Después de los trabajos propuestos en el denominado "Proceso Bolonia" los europeos han seguido trabajando en la innovación y armonización curricular reuniéndose en Leiria (Portugal), Helsinki (Finlandia), Malmö y Goteborg (Suecia), Salamanca, Niza; Barcelona y Berlín, entre otros.

Las reformas que se están produciendo son tan profundas que van a suponer una revisión trascendental de todas las carreras universitarias en cada uno de los países de la Unión Europea y de allí se difundirán a otras áreas económicas de terceros continentes y en especial para Iberoamérica como se destaca en la primera reunión de Ministros de Educación de la Unión Europea, Latinoamérica y Caribe celebrada en París el 2 y 3 de noviembre 2000 y en la reunión de Torino, el 17 de noviembre 2000 , por los presidentes, rectores y representantes de aquellas universidades y otros "encuentros» como el que tuvo lugar en Santiago de Chile en octubre de 1998 y en Montevideo en el año 2000.

Ya hemos destacado que la idea principal de la que parte el inicio de estas reformas son el modernizar y homogeneizar las titulaciones para facilitar el reconocimiento mutuo entre los países miembros, flexibilizarlas para poder hacer más fácil su adaptación a las necesidades de la sociedad y facilitar la movilidad de los graduados, a la vez que hacer frente a la necesidad de mejores técnicos y científicos que escalen de nuevo a las cumbres de las perdidas cotas europeas que fueron mantenidas hasta hace, todavía, poco tiempo. Estas reformas suponen, también, la utilización común de un sistema de créditos que sea uniforme para toda Europa, similar a los que se están aplicando en los conocidos programas Sócrates/Erasmus, hasta ahora validos sólo para países miembros de la Unión.

Así pues, se pretende la acreditación homogeneizada de los distintos programas de estudio y que, estos, vengan acompañados de la armonización del contenido y fe pública de esta y de su calidad. 
Acreditar un programa académico implica dar cuenta y ser conscientes acerca de su misión o, lo que es lo mismo, saber lo que pretende lograr con su programa académico de formación profesional para los estudiantes de las distintas carreras universitarias. Ha de controlar sus resultados, debiendo demostrar que posee un programa y recursos para llevar a cabo sus objetivos y definir los procedimientos utilizados para evaluar y contrastar los resultados $\mathrm{y}$, como en toda programación, ha de disponer de un plan a ejecutar en el caso de que sea necesario corregir las deficiencias que puedan encontrarse o introducir nuevos aportes de conocimiento científico.

Ni que decir tiene que el papel que hoy deben cumplir los profesionales que formamos en las distintas universidades viene determinado dentro de un marco legal, social y económico establecido según las nuevas exigencias tanto nacionales como internacionales. Es decir, debemos hacer frente a la globalización y su impacto ante la integración económica entre países tan diversos. Caminamos hacia un profesional globalizado que precisa, debido a la competitividad, de un perfeccionamiento continuo que solo puede alcanzarse a través de la especialización y con la continua atención a los estándares de formación profesional existente en las universidades del resto del mundo. Se precisa para ello, el intercambio y las relaciones, tanto personales como institucionales, de nuestras universidades, de sus docentes y de nuestros universitarios. Relaciones con organismos nacionales e internacionales y líneas de investigación consolidadas y emergentes, financiadas con fondos tanto internos como externos y, sobre todo, el fortalecimiento de la calidad en la formación tanto universitaria como pre y postuniversitaria.

En este contexto, nuestras universidades han de ser capaces de diseñar un plan de formación flexible y práctico, basado no sólo en lo que se ha tenido como estructura tradicional, sino que, también, ha de contener disciplinas útiles relacionadas con las denominadas troncales con visiones que permitan apreciar la importancia de otras materias, sin descuidar, por supuesto, una formación estructural homogénea dentro del perfil pretendido.

El currículo, por su parte, es un elemento dialéctico, investigativo y de desarrollo permanente, que sirve como instrumento o medio para conseguir unos objetivos, propósitos, metas y fines futuros que estamos pretendiendo. El cambio curricular debe promover la definición de competencias transversales en todos los planes de estudios de formación general, paralela a la inicial, y también una mayor flexibilidad en la formación de la especialidad, permitiendo una cierta 
movilidad horizontal de los estudiantes, como también su avance hacia módulos curriculares que vinculen más ágilmente los estudios de la licenciatura con los estudios de postgrado, en lo que debe ser el inicio implícito de una formación continua.

Con estos programas se conseguirá el enriquecimiento personal, cultural y de comunicabilidad del estudiante como desarrollo armónico de sus capacidades y de sus virtudes con el soporte de una formación general sólida que será completada con su formación en la especialidad.

No decimos que la concreción de esta armonización curricular sea un proceso fácil o simple, sino al contrario. La gran mayoría de los currículos de los programas de formación existentes son tan rígidos como arcaicos y su supuesta flexibilidad, reflejada en una que otra asignatura optativa, es muy relativa. Los actuales sistemas obligan, además, a una especialización muchas veces prematura, sin permitir alternativas para diversificar el proceso formativo. Muchas veces, además, determinan salidas profesionales únicas sin existir calificaciones profesionales de grado medio.

Para la ejecución del cambio de la armonización curricular es necesario que los claustros académicos dejen sus repartos personales y egoístas de materias y tengan la voluntad y apertura suficiente para convenir los principios y conceptos generales de lo que debe ser una formación de licenciatura, práctica, flexible y modularmente construida. Esto es fundamental para avanzar hacia la toma de decisiones de reforma y modernización de la armonización curricular tanto como para conseguir las adecuaciones necesarias en la reglamentación interna de nuestras universidades. Esto es así porque la actual normativa, las normas y reglamentos existentes, pueden constituir un obstáculo para la flexibilidad y la movilidad intra e interinstitucional de la que hemos hablado y que se quiere favorecer. En consecuencia, es importante analizar la actual normativa y los reglamentos y replantearlos para conseguir su armonía con un modelo curricular que pueda aprovechar las ventajas que nos plantea la modernidad en la formación superior para llegar a igualarnos con los países más desarrollados.

\section{CRITERIOS EN EL DISEÑO E IMPLANTACIÓN DE UN PROCESO DE FLEXIBILIZACIÓN CURRICULAR}

Hemos de conseguir una correspondencia explícita y positiva entre los grados intermedios y los títulos superiores profesionales y que estos sean flexibles, homologables y comparables con otros pro- 
fesionales universitarios extranjeros con los que ya estamos empezando a competir. El no propender a modernizar nuestros sistemas de enseñanza y formación y el no posibilitar que sean comparables y mayores salidas o "pasarelas curriculares» implica limitarlos en sus legítimas aspiraciones.

Uno de los mayores retos que tienen nuestras universidades consiste, posiblemente, en configurar su oferta académica de grado, postgrado y doctorado de manera sistemática y efectiva, de acuerdo, no sólo con la competencia de los profesionales comunitarios y de terceros países, sino, además, con las demandas de trabajo del entorno, a corto, medio y largo plazo.

La segmentación de esas demandas suele encontrarse en las expectativas del mercado laboral y del sistema productivo; en las necesidades de desarrollo científico y tecnológico y en los problemas del contexto político e institucional, como venimos advirtiendo, derivados de los procesos de globalización e internacionalización de la economía. De ahí, las continuas reuniones y congresos que vienen produciéndose, al respecto, dándose cita para intentar responder a cuestiones relacionadas con los procesos de gestión de la demanda y de la oferta académica y debatir las prácticas que utilizan las distintas universidades para definir e igualar sus líneas prioritarias de desarrollo. En resumen, se trata de definir, seguimos repitiendo, las tendencias y desarrollo en los estudios de grado, postgrado y doctorado en nuestras universidades y su convergencia y armonización curricular con las del resto de la comunidad sin olvidar la gestión de la innovación tecnológica y social.

Para alcanzar estos objetivos, para su diseño e implantación, son necesarios ciertos criterios mínimos, entre los que podríamos destacar el hecho de que la flexibilización y armonización curricular debe ser un objetivo general en un contexto de cambio planificado. Los cambios de este proceso de flexibilización curricular tienen que considerar las demandas de una formación inicial y no terminal de los estudiantes. Los currículos propuestos, producto de la flexibilización curricular, deben contemplar una formación general y especializada con obligaciones adecuadas y pertinentes y los productos curriculares de las acciones de flexibilización han de definir las estructuras y los modos de articulación de y entre los diferentes programas de la institución, definir los niveles de autonomía de los estudiantes en el contexto de la oferta educativa de la institución y, además, han de considerar procesos de información y orientación tutorial de la oferta educativa por parte de cada Universidad. 
La flexibilización curricular ha de vincularse de manera profunda al proceso de modernización de la Educación Superior y así, esta flexibilización se circunscribiría a hacer sus planes y programas homologables en lo posible, tanto en sus estructuras como en sus ofertas y productos académicos con los actuales planes y programas europeos más avanzados y norteamericanos y, así mismo, en lo posible, con las universidades iberoamericanas.

Frente a un trabajo académico orientado por planes de estudios rígidos e inflexibles, obligatorios, con excesiva carga académica y desmedido énfasis hacia cursos teóricos, la flexibilización curricular demandaría en su forma operativa una reformulación de este diseño. Se propone una ruta alternativa a la concepción lineal y rígida de los estudios profesionales rompiendo así con el sistema de materias y cursos divididos y obligatorios. El cambio curricular, por consiguiente, ha de representar la concreción de una gama de opciones para la formación profesional de los estudiantes.

\section{LA DESCONEXIÓN SECULAR ENTRE LA UNIVERSIDAD Y LA EMPRESA}

Otro de los problemas a considerar es el de la desconexión entre la Universidad y la Empresa que ya quedó reflejada en el Tratado de Roma de 1957, en el que se trataba de forma muy superficial el tema de la política educativa y sólo se reflejaban algunos aspectos de la formación profesional en los artículos 57; 118 y 128 del mismo, en contraposición al desarrollo que se realizaba en aquel de otros temas de la economía real. Posteriormente, los Ministros de Educación de la Comunidad Europea se reúnen por primera vez en 1971, y a continuación de la primera ampliación de 1972 y el informe del Prof. Henri Janne de 1973, se comienzan a sentar las bases para los futuros programas educativos comunitarios, sobre la base de fijar como un derecho de todos los ciudadanos de la comunidad, el de la enseñanza, reconociendo la necesidad de sintonizar más estrechamente los sistemas de enseñanza, formación y empleo, manteniendo diversidad de los sistemas educativos de los Estados miembros pero estableciendo sistemas de cooperación bajo la responsabilidad de cada uno de los Estados.

En 1976 el Comité de Educación de la CEE redactó un informe que propugnaba un cambio importante en la política de formación. La idea básica era que la política educativa debería adoptar las medidas necesarias para prevenir las necesidades futuras, en vez de actuar para 
remediar los problemas ocurridos en el pasado, concretándose en una serie de proyectos de los Estados miembros, que permitieran la adaptación a las condiciones especiales de cada país intercambiando experiencias en los temas de formación. Las áreas prioritarias de estos proyectos fueron: la reforma de la formación profesional; el desarrollo de nuevos modelos de orientación profesional y educativa, y los sistemas experimentales que alternaran el trabajo con la enseñanza superior y la movilidad en otras Universidades de los estudiantes.

\section{UN MOMENTO PARA EL CAMBIO}

Evidentemente hay estudios que son unos más que otros adecuados para homogeneizarse y partir con el proceso de flexibilización. Las Facultades técnicas y de ciencias son las que, por su naturaleza y la convergencia curricular con las ciencias básicas, presentan módulos de formación que deben y pueden ser similares en el conjunto de las Universidades, no sólo por la necesidad de esta amornización, sino, también, porque los profesores intentan y aceptan más la similitud de programas de asignaturas de otras universidades avanzadas con las suyas propias.

Fue con el "Libro Blanco de la Comisión de las Comunidades Europeas» sobre "Crecimiento, Competitividad y Empleo", publicado en 1993 el que pone de relieve la necesidad de muchos de estos cambios, aclarando que lo que se plantea son los «retos y caminos para entrar en el siglo XXI». La necesidad y motivos para estos cambios se recogen en este interesante documento sintetizándolos en los siguientes motivos:

1. Geopolíticos, como la aparición de nuevos países competidores con capacidad para aplicar los progresos técnicos más avanzados.

2. Demográficos, caracterizados por el envejecimiento de la población, la transformación de las estructuras familiares y los movimientos migratorios de población.

3. Técnicos, sustentados por la revolución de las tecnologías de la información que afecta a la industria, el empleo y la competencia con incidencia en los sectores económicos donde la información pasa a ser un factor clave.

4. Financieros, apoyados por la interdependencia de los mercados, como consecuencia de la libertad de movimiento de capitales y la utilización de las tecnologías de la información. 
Otra consecuencia negativa de estos cambios es el nivel de paro en Europa que ha llegado a afectar a cerca de 20 millones de ciudadanos y pone de relieve que los sistemas de empleo de los países de la Unión han quedado obsoletos. La recuperación exige nuevos enfoques y planteamientos en relación con las actividades del trabajo y el empleo que ha de estar soportado por las nuevas tecnologías de la información, que están afectando a los métodos de producción, a las pautas de consumo y, en definitiva, a la sociedad en general. Todo esto implica una nueva revolución, tan importante como fue la propia revolución industrial, diferenciándose de esta en que en vez de ser el motor del desarrollo, el capital financiero, lo será el capital humano o lo que es lo mismo, la educación y la formación van a ser las palancas claves para desarrollar la competitividad de las empresas, la creación de empleo y la integración de los individuos en la sociedad.

Los nuevos planteamientos pretenden que la formación y la enseñanza afecten a las personas no sólo en el periodo de su juventud, sino a lo largo de toda su vida y ello exige un nuevo planteamiento de la enseñanza y la formación donde existan fórmulas integradoras de las instituciones académicas, los organismos de formación y de las propias empresas y de su financiación.

\section{EL PROYECTO DE MODELO DEL ESPACIO EUROPEO DE LA EDUCACIÓN SUPERIOR}

Hemos destacado que este proyecto comienza con la determinación del denominado "Espacio Europeo de Educación Superior» y pretende conjuntar una serie de modelos educativos existentes, optimizándolos. Este concepto que se conoce como el «Espacio Europeo de Educación Superior" se inicia con la "Declaración de la Sorbona» de 1998 y es un proceso que se consolida con la Declaración de Bolonia de 1999 y, continúa con la Cumbre de Jefes de Estado de Praga en el 2001 y la de Cumbre de Barcelona de 2002 para reafirmarse en la Conferencia de Berlín de 2003.

El 25 de Mayo de 1998, los Ministros de Educación de Francia, Alemania, Italia y Reino Unido firmaron en la Universidad parisiense de la Sorbona una declaración instando al desarrollo de un «Espacio Europeo de Educación Superior», como un proceso político de cambio a largo plazo de la enseñanza superior en Europa. Un año después, el 19 de Junio de 1999, los ministros responsables de la educación superior de 29 países europeos firman en Bolonia un 
acuerdo donde se fijan los objetivos más importantes para la construcción del Espacio Europeo de Educación Superior.

"Las razones para la creación del Espacio Europeo de Educación Superior no olvidan la influencia de la educación y la cultura en la formación de una Europa unida: Europa no es sólo la del Euro, de los bancos y la Economía: debe ser una Europa del conocimiento también. Debemos reforzar y construir la dimensión intelectual, cultural, social y técnica de nuestro continente». Esta Declaración destaca sobre todo, la importancia de capital humano para la construcción de la Europa del futuro: «Una Europa del conocimiento es ampliamente reconocida como un factor irremplazable para el crecimiento social y humano y como un elemento indispensable para consolidar y enriquecer a los ciudadanos europeos, capacitándolos con las necesarias competencias para afrontar los retos del nuevo milenium», asignando a las Universidades un papel fundamental de los cambios que se vincula al fomento de la movilidad y el empleo como medio de desarrollar los niveles de competencia y excelencia. "Los estudiantes deben tener acceso a una diversidad de programas, con la oportunidad de estudios multidisciplinares incluidos, al desarrollo de la capacitación en idiomas y las habilidades para usar las nuevas tecnologías de la información». Tampoco olvida la necesidad del contacto de los estudiantes con entornos culturales y económicos diferentes al suyo propio: «Tanto a nivel de graduados como de postgraduados, los estudiantes deben ser animados a realizar al menos un semestre en universidades de fuera del propio país».

Posteriormente la «Declaración de Bolonia» concreta y establece las ideas marco para la creación del «Espacio Europeo de Educación Superior" basándose en los principios de calidad, diversidad, movilidad y competitividad y buscando dos aspectos trascendentales: el incremento del empleo en la Unión Europea y la conversión del «Sistema Europeo de Formación Superior» en un polo de atracción para estudiantes y profesores de otras partes del mundo.

La «Declaración de Bolonia» de 1999 tiene, sin embargo, tan solo un carácter político, enunciando una serie de objetivos a cumplir y unos instrumentos para conseguirlos, sin fijar normas ni obligaciones jurídicamente exigibles. Es por esto que son los países que se adhirieron los que tendrán que imponer los instrumentos diseñados para alcanzar los objetivos propuestos. Flexibilización curricular, homogenización y modernización de los estudios; convenios con otras Universidades nacionales e internacionales; estancias en instituciones extranjeras, en definitiva, un cambio estructural y construcción conjunta dentro de este cambio planificado. Se pretende, en 
definitiva, la formación de profesionales con capacidad para plantear y resolver problemas, de acuerdo con las nuevas realidades de las demandas sociales y educativas con el propósito de lograr un mayor enriquecimiento interdisciplinario entre los mismos, a fin de elevar la capacidad de adaptación a las transformaciones previsibles de la demanda social a la Educación Superior.

Los instrumentos propuestos para alcanzar los objetivos que venimos destacando como que los estudios universitarios tengan mayor transparencia y similitud entre los diferentes países europeos son, entre otros:

1). La generalización del Sistema del "Crédito Europeo" denominado European Credit Transfer System (ECTS) como elemento clave del modelo Europeo. El crédito ECTS representa, en forma de valor numérico, la carga de trabajo de un estudiante para superar un curso académico anual, que se valora en 60 ECTS. Por tanto, un semestre equivale a 30 ECTS y un trimestre a 20 ECTS. En dicha valoración de la carga de trabajo, necesaria para completar un curso académico, se incluyen las clases magistrales, trabajos, seminarios, periodos de prácticas, horas de estudio, exámenes, y todo aquello que el alumno necesite para adquirir los conocimientos y competencias necesarias para superar las materias de un curso académico. Para estimar el valor en horas de trabajo de un crédito europeo se supone que un alumno que estudia a tiempo completo dedica 40 horas de trabajo a la semana, lo que, multiplicado por las 40 semanas de un curso académico, equivalen a unas 1.600 horas de trabajo por curso. Dado que dicho curso tiene un valor máximo de 60 créditos, el valor de un crédito en horas de trabajo está en torno a las 25-30 horas. Muchas universidades europeas han venido aplicando un sistema de transferencia de créditos, entre los alumnos que han realizado algún tipo de intercambio con programas de movilidad de estudiantes, como los programas Erasmus o Sócrates. Este sistema de transferencia de créditos ha venido funcionando desde 1989 y de lo que ahora se trata es de generalizar su aplicación, no sólo para los estudiantes que realizan intercambios sino también para todos los estudiantes de la Unión Europea. El crédito europeo es, una medida de los conocimientos adquiridos por los alumnos así como un sistema que permite homologar dichos conocimientos entre las instituciones universitarias. De esta forma, el estudiante puede adquirir los conocimientos y competencias que desee a lo largo 
de toda su vida, completando los procesos de formación que estime necesarios.

Este sistema de créditos ECTS permite, como decimos, valorar los conocimientos y competencias de los alumnos adquiridos en distintos sistemas de enseñanza y ofrece una información clara sobre los progresos adquiridos en su formación facilitando la movilidad de los estudiantes. Sin embargo, su aplicación práctica exige un nuevo sistema de enseñanza donde el papel del profesor se transforma en el elemento esencial para ofrecer el apoyo y la asistencia necesaria al alumno para el desarrollo con éxito de su trabajo.

2). Adopción del denominado «Suplemento al Título o Diploma» que facilitará la información necesaria sobre la formación recibida por el alumno de cara a su reconocimiento por otras instituciones académicas o para facilitar su empleo en las empresas. El Suplemento al Diploma es la herramienta fundamental para facilitar el reconocimiento de títulos en la Unión Europea. La información que comprende ofrecerá los datos más relevantes sobre el estudiante y su formación adquirida, nivel y duración del programa realizado, contenidos con programa de estudios detallado y calificaciones obtenidas (expediente académico en créditos europeos), así como su titulación y la Institución que lo expide; información sobre la función de la titulación, etc.

El «Suplemento Europeo al Título» es, en definitiva, un documento de información unificado y personalizado para el titulado universitario sobre los estudios cursados, su contexto nacional y las competencias y capacidades profesionales adquiridas en su formación a lo largo de su vida en las diferentes instituciones universitarias de la Unión Europea.

3). Adopción de una estructura de la enseñanza basada, no en uno, sino en dos ciclos académicos. El proyecto diseñado por la Declaración de Bolonia establece una titulación básica de grado, que dará lugar a un título con cualificación profesional en el mercado laboral europeo, y un segundo nivel, de postgrado, que se podrá cursar después de haber obtenido el grado y que permitirá obtener las titulaciones de Master y/o Doctorado. Posteriormente, la declaración de Berlín especificó que cada uno de estos títulos deberán tener una orientación diferente para acomodarse a las distintas demandas de los individuos y a las necesidades académicas y laborales que 
demanden los mercados. Pero además indica que el título de segundo nivel o postgrado servirá para dar acceso a los estudios de doctorado. La duración de los estudios de grado, o primer nivel, se fijó en relación con la cantidad de créditos ECTS a obtener que habrían de ser entre 180 y 240 , que corresponderían a tres ó cuatro cursos académicos.

Estas titulaciones, además, deberán diseñarse en función de unos perfiles profesionales con perspectivas nacionales y europeas y con unos objetivos que han de hacer mención expresa a las competencias genéricas, trasversales y específicas, es decir a los conocimientos, capacidades y habilidades que se pretenden alcanzar. Cada Gobierno nacional ha de aprobar las directrices generales de cada titulación y el cuadro de títulos de grado. Por su parte, las universidades elaborarán los planes de estudios que deberán ser homologados por la autoridad competente. Cuando se realice el nivel de postgrado habrá de distinguirse entre las enseñanzas conducentes a los Masteres y las que se encaminen a los Doctorados, sin que por el momento se haya definido si estas serán secuenciales o alternativas. Los objetivos de formación del Master serán más específicos que los de Grado y deberán estar orientados hacia una mayor profundización, posibilitando un desarrollo académico disciplinar e interdisciplinario de especialización científica, de orientación a la investigación o de formación profesional avanzada. La obtención del título de Master requerirá, por su parte, haber completado entre un mínimo de 60 y un máximo de 120 créditos ECTS lo que implica la posibilidad de diseñar los estudios de postgrado en uno o dos años.

\section{LAS DIFICULTADES EN LA APLICACIÓN DEL PROYECTO}

La opinión política en general pretende la implantación del modelo europeo de enseñanza superior que se diseñó en la Declaración de Bolonia sin que ocurra lo mismo con las instituciones universitarias, principales actores del proceso, que en muchos casos destacan los problemas específicos y concretos que supone la pretendida convergencia en el terreno de la educación superior. Estos problemas se derivan, no por rechazo a los principios del nuevo modelo europeo, sino a la distancia que separa a los países, incluso a las Universidades, en función de las circunstancias de partida tan distintas. 
Hoy por hoy, las diferencias entre las situaciones actuales de los sistemas universitarios de los diferentes estados europeos hace difícil identificar un verdadero "modelo europeo" que implicaría, en cierta medida, adaptarse por sus muchas coincidencias con el sistema universitario anglosajón. Así, por ejemplo, la aplicación de sistema de créditos europeos ECTS, representa el valor numérico del trabajo que el estudiante debe realizar para superar las materias de un curso académico, mientras que en España, el sistema de créditos actual se basa en el número de horas lectivas de las materias por lo que el proceso de convergencia a realizar en España para adaptarse al sistema de "créditos europeo» será más complejo que para otros países, como los anglosajones, con sistemas más análogos.

Igualmente sucede con la estructura de las enseñanzas. Los países que, tradicionalmente, tenían una estructura de grado y postgrado muy clara, les costará menos adaptarse al modelo que aquellos otros, como el nuestro, que tienen diversos niveles de graduados (Diplomados y Licenciados) y una escasa implantación del nivel del postgrado o Master.

Otro inconveniente a superar ha sido, también, el marco legal donde éste ha de desarrollarse. Así, en algunos países, las reformas necesarias se consiguen con pequeñas correcciones legales, que han hecho compatibles los cambios con la situación anterior a los mismos, mientras que en otros los cambios que exige la aplicación del modelo europeo se han buscado aplicar reformas más profundas de las estructuras universitarias, las resistencias al cambio son mayores.

El problema de la implantación de estas reformas y criterios no es solamente teórico. Un cambio tan fundamental precisa trasladar a las normas jurídicas de cada país las reformas necesarias para realizar este proceso. Así, en el «Comunicado de Praga» del año 2001 ya se introducen algunas líneas de actuación adicionales, tales como la implantación del aprendizaje a lo largo de toda la vida lo ha venido a conocerse por «Long Life Learning».

Igualmente se propugna por el establecimiento de ciertos sistemas de garantía de la calidad y de mecanismos de certificación y acreditación para generar una mayor aceptación y atracción de los títulos hacia el Espacio Europeo de Educación Superior y el papel activo de las universidades y de sus miembros en este proceso de convergencia. Posteriormente, un año más tarde en la Cumbre de Jefes de Estado de Barcelona de 2002, se acentúan los aspectos relacionados con la movilidad en los ámbitos de la educación, la investigación y la inno- 
vación, así como la eliminación en los obstáculos normativos y administrativos al reconocimiento profesional.

Finalmente hemos de destacar, la Conferencia de Ministros de Educación Superior celebrada en Berlín el 19 de Septiembre de 2003 donde se matizan algunos de los aspectos establecidos en anteriores Conferencias de Ministros o Cumbres de Jefes de Estado, estableciendo fechas para la realización de determinados objetivos. Así, se estableció este mismo año de 2005 para el establecimiento de los sistemas nacionales de acreditación de la calidad, la puesta en marcha de la estructura de los estudios en dos ciclos principales (grado y postgrado); la emisión a los graduados del Suplemento al Título o Diploma, y el reconocimiento de los títulos y periodos de estudio, cuyos objetivos serán revisados en la próxima reunión de Bergen (Noruega) en mayo de este mismo año.

\section{LA IMPLANTACIÓN DEL MODELO EUROPEO EN ESPAÑA}

La adaptación de nuestra enseñanza al modelo europeo comienza cuando en febrero de 2003 el Ministerio de Educación, Cultura y Deporte, hizo público un «Documento Marco» sobre la integración del sistema universitario español en el Espacio Europeo de Enseñanza Superior. En dicho documento se recogían las principales referencias que la Ley Orgánica de Universidades 6/2001 (LOU) establecía para la integración de nuestro sistema universitario en el futuro Espacio Europeo de Enseñanza Superior.

En el sistema universitario español, la lista de las tareas, a realizar por las instituciones integrantes del sistema de enseñanza superior, presentaba un proceso desigual. Es decir, mientras que la adopción del sistema europeo de créditos ECTS contiene los elementos necesarios para su futuro desarrollo, la adaptación de las enseñanzas y títulos oficiales universitarios no está prácticamente definida lo que implica la necesidad del oportuno desarrollo normativo, además de la elaboración de ciertos proyectos que permitan establecer las directrices generales propias de cada titulación en particular.

Ha de destacarse, igualmente, otro de los contenidos que ha desatado un debate dentro de la comunidad universitaria cual es la aplicación de los principios de calidad que se contienen implícitos en las reformas para alcanzar el «Espacio Europeo de Educación Superior».

La promoción de la evaluación de la calidad y de la acreditación a nivel institucional, nacional, regional e internacional se hace hoy im- 
prescindible, en un contexto de internacionalización creciente y con el fin de crear la confianza necesaria en la evaluación, el aseguramiento de la calidad y la acreditación. Es por ello necesario identificar las organizaciones, los instrumentos e iniciativas existentes, así como difundir sus experiencias, procesos y resultados para promover su mutuo reconocimiento e impulsar el desarrollo de nuevos esfuerzos.

En España, los mecanismos y procesos de evaluación, certificación y acreditación fueron encomendados a la Agencia Nacional de Evaluación de la Calidad y Acreditación (ANECA) y han suscitado polémicas y recelos ante una práctica que, en determinados aspectos, no tenía los elementos de transparencia necesarios para ser entendida por la comunidad universitaria. En cierto sentido podríamos decir que, si bien los objetivos de la implantación del Espacio Europeo de Enseñanza Superior, son compartidos, no gozan de este mismo consenso general los mecanismos y medios que se han arbitrado para alcanzar dichos fines.

Hemos de destacar, en primer lugar, la falta de la financiación oportuna para abordar las transformaciones que se necesitan realizar en nuestro país. Por ejemplo, los proyectos encaminados a la realización de los estudios previos para el diseño de las nuevas titulaciones en el campo de las Ciencias Económicas y Empresariales no fueron concedidos en la primera convocatoria, al parecer, por problemas presupuestarios. Es por ello que las preocupaciones de las universidades sobre la falta de financiación para la implantación del futuro modelo europeo ha sido un factor de desconfianza en lo que se refiere a la implantación y el desarrollo del proceso de adaptación de los cambios para la convergencia universitaria con los países más evolucionados de la Unión Europea.

Insistiendo en ello, otro de los factores que han contribuido a la desconfianza en los comienzos de esta reforma universitaria ha sido la actuación de la propia ANECA en relación con la acreditación del profesorado contratado. La aplicación de criterios poco definidos y operativos a la hora de valorar los méritos de los profesores para optar a las nuevas fórmulas de contratación ha suscitado recelos que se están haciendo más evidentes a medida que se acerca el año 2006 en el que finaliza el periodo transitorio establecido por la propia LOU.

No se ha producido el conveniente acuerdo de todos los responsables en el proceso para lograr el necesario grado de consenso, tanto en los objetivos finales como en las medidas necesarias para conseguirlos. Las declaraciones de la Conferencia de Rectores de la Universidad Española, de los partidos políticos y de las administra- 
ciones autonómicas, han puesto de relieve las discrepancias entre uno con los otros y con el diseño de la propia política universitaria del Ministerio.

Resumiendo, en lo que a España concierne y se reconoce es que, en definitiva, la convergencia hacia el "Espacio Europeo de Enseñanza Superior» es una meta deseable para lograr una mejor formación de los futuros dirigentes de nuestro país, pero la trayectoria hasta alcanzar esta meta se enfrenta a importantes retos y dificultades que es necesario abordar dejando a tras los egoísmos y repartos de poder indeseados, con un mayor realismo, mayores medios e ilusión si se desea llegar a la fecha del 2010 con los objetivos alcanzados y posibilidades reales de éxito.

\section{LA PROYECCIÓN EN IBEROAMÉRICA DE LA CONVERGENCIA CURRICULAR Y CONCLUSIONES}

Todos los temas sobre la modernización de la enseñanza, su homogeneización y armonización curricular, confrontación de experiencias, etc que pretende el proyecto del «Espacio Europeo de Enseñanza Superior», seguramente, una de las principales actuales preocupaciones de los responsables universitarios, vienen tratándose, como decimos, en continuos congresos y reuniones. Queremos destacar por lo que nos concierne por nuestra relación con iberoamérica, las «Declaración de Valparaíso» y las conclusiones del Seminario Internacional «Innovación y Armonización Curricular: Confrontación de Experiencias, Perspectivas de Colaboración" en el que, precisamente, se perfilan los hitos principales necesarios para aquella tan deseada armonización curricular promoviendo la convergencia del sistema universitario de ciertos países iberoamericanos, homogeneizándonos con las transformaciones que tienen lugar en los países de la Unión Europea entre los que se encuentra España.

Ha de impulsarse en las universidades iberoamericanas, la adopción de un sistema de créditos compatible con el de las universidades europeas y entre ellas mismas. Reforzar las relaciones de las universidades con los actores externos relevantes para el desarrollo y el mejoramiento de la oferta formativa, estimulando acuerdos recíprocos de doble titulación, tanto en los niveles de grado como de postgrado, con centros académicos tanto europeos como americanos de prestigio, a la vez que fomentar la movilidad de estudiantes, profesores y personal de apoyo, todo ello en pos del desarrollo del conocimiento y para el intercambio y posicionamiento competitivo de las distintas titulaciones. 
Los Estados deben readecuar las normas relativas a la duración de las carreras universitarias como indicador principal para asignar niveles en grados y la implantación de un nuevo currículo de formación de los distintos grados. Así, una primera alternativa es analizar y flexibilizar los currículos evaluando la posibilidad de establecer concordancias y/o "pasarelas» o trayectorias entre las distintas carreras, revisando los criterios de homologación y convalidación de los programas de asignaturas y definiendo un sistema de créditos semejante al europeo como referencias en la formación para el establecimiento de niveles dentro del proceso de homologación necesario.

Es conclusión importante tener presente que los cambios de índole político, social y especialmente en área de desarrollo económico, han propiciado variaciones importantes en los sistemas universitarios; aspectos que ha tenido una influencia importante en la generación de una nueva filosofía de la educación que se está concretando en estos proyectos. Desde esta perspectiva, se destaca la necesidad de orientar la formación del profesional universitario hacia el manejo integral del proceso educativo dentro del contexto y necesidades, relacionadas con las distintas necesidades de la sociedad. Tenemos, en definitiva que ofrecer más de lo que estamos dando y tenemos que servir a más a los estudiantes a los que estamos formando.

Por ello han de abrirse espacios para el intercambio de conocimientos docentes y realización de investigaciones conjuntas que potencien la formación integral y constante, tanto del profesorado como del nuevo profesional. La aparición y desarrollo de la informática ha venido acompañada, principalmente en Estados Unidos y Europa, de un cambio en la orientación de la formación. Esto es debido a la necesidad de garantizar la eficacia, seguridad y utilización correcta de los conocimientos, hoy puestos al alcance de todos por este medio y todos los grandes sistemas de comunicación y acceso a la información que ha de dirigirse, también, hacia los elementos centrales del proceso formativo.

Se destaca y se hace preciso la compatibilización curricular en relación al peso específico o tiempo de dedicación o carga horaria de las áreas en que se agrupan las asignaturas de cada especialidad, así como cuales han de ser los contenidos mínimos para cada una de las áreas en que se agrupan las asignaturas de cada especialidad.

Los conocimientos deben estar cimentados por contenidos relevantes e integrados de manera que lo capaciten gradualmente para un ejercicio idóneo de la profesión en los diferentes campos del de- 
sempeño profesional. Gestionar y liderar su puesto de trabajo o actividad laboral, en forma efectiva y eficiente, garantizando su rentabilidad económica y social; así como la creación de empleos, incluso de empresas lo que requiere de conocimientos sobre la gestión de recursos económicos, físicos y del talento humano, al igual que sobre la planeación, diseño, ejecución y validación de procesos. Identificar, caracterizar, intervenir y priorizar la resolución de problemas relacionados con su quehacer; tomar decisiones, trabajar en equipo en forma interdisciplinaria y delegar, utilizando en forma adecuada los sistemas de información y tener la destreza de identificar y seleccionar la información relevante para la solución de la problemática propia de su profesión, manteniendo, siempre, como venimos repitiendo, un proceso de autoformación y formación permanentes.

Se pretende, en definitiva que el nuevo graduado practique ciertos valores y filosofía de su profesión, observando valores éticos como la confianza, el respecto por la diferencia, la responsabilidad social, el compromiso, la honestidad, la transparencia, la equidad y la justicia. Resulta esencial destacar el carácter participativo que por lo general requiere todo tipo de actividades profesionales por lo que se debe superar la tendencia que poseemos a trabajar individualmente. Esta filosofía nos llevará a establecer vínculos de cooperación. A potenciar las redes y la movilidad entre nuestras universidades e instituciones y a fomentar la convergencia de nuestros sistemas de educación superior, compartiendo nuestros valores comunes profundamente arraigados y aceptando los nuevos que los mejoren.

Otra de las conclusiones es la conciencia de los efectos negativos del aislamiento y de la incompatibilidad así como de los grandes beneficios que los cambios concordados y las acciones conjuntas pueden suponer tanto para los alumnos como para el profesorado como se ha reiterado numerosas veces y tanto se destacó en la «Declaración de Florianópolis» y la «Declaración Torino».

Son varias las líneas de acción a realizar respecto a la enseñanza universitaria iberoamericana. Entre estos objetivos hemos de destacar la necesidad de la homologación convergente de los distintos sistemas de educación superior de Europa e iberoamérica para propiciar su posible comparabilidad y compatibilidad. Esto precisa desarrollar normas, criterios, instrumentos y mecanismos que, de manera clara y precisa, definan los objetivos y procesos de la educación superior de estos países. Que se produzcan los cambios que sean necesarios en el interior y fuera de las Universidades para mejorar el aprendizaje y favorecer la competencia de los estudiantes y gradua- 
dos, así como que se propicie el tránsito de los estudiantes entre sus diversos ciclos de estudio y las instituciones de los ambos continentes.

Hemos destacado, también, la necesidad de renovación de los curricula a fin de incrementar su contribución a las políticas de desarrollo de los distintos países, lo que implica, entre otras cosas, impulsar la revisión de los contenidos, organización y lógicas de estos para diseñar otros que sin menoscabo de la calidad, sean más flexibles, breves y pertinentes.

En el contexto futuro de internacionalización creciente, es imprescindible crear confianza en la evaluación, el aseguramiento de la calidad y en la acreditación. Es por ello por lo que es necesario identificar las organizaciones, los instrumentos e iniciativas existentes, así como difundir las experiencias, procesos y resultados de cada uno de los países y promover su mutuo reconocimiento y aplicación de estos instrumentos e iniciativas en las instituciones y programas de ambas regiones y de forma comparada para asegurar su uso transparente y propiciar la movilidad.

Es esta, la movilidad académica otro componente esencial del curriculum de estudiantes que representa una fuerza importante que estimula la renovación académica y promueve el acercamiento cultural. Se precisa extender sus posibilidades, multiplicando las modalidades, que fortalezcan las interrelaciones entre países y con otros aspectos de la vida universitaria, laboral y social internacional. Han de establecerse redes y lazos de cooperación transcontinentales; renovar los planes de estudio tanto en relación con el acceso más selectivo a la universidad; mejorar sus contenidos, orientación y enfoque pedagógico y reconocer con las garantías necesarias las titulaciones y los créditos de los países homologados.

En la reunión celebrada por los presidentes, rectores y representantes de las universidades de Europa e iberoamérica el 17 de noviembre del 2000 en Torino, se trató de la reestructuración convergente de los sistemas de educación superior de estos países promoviendo un esquema regional para las titulaciones de educación superior en cada región, que permita su comparación y compatibilidad y adoptando sistemas compatibles para la acumulación y transferencia de créditos académicos, inspirados en las experiencias del ECTS y diferenciando los estudios en dos niveles,

Igualmente se trató de la renovación de los curricula con el fin de incrementar su contribución a las políticas de desarrollo, diseñando, 
como ocurre en la Unión Europea, unos curricula más flexibles (con sistema de créditos y opciones alternativas) y organizados en programas secuenciales más cortos, con la posibilidad de acceder al mercado de trabajo al completar el primer nivel.

Un aspecto que nos interesa particularmente por su relación con nuestra universidad es el planteamiento de diversificación sobre las distintas condiciones de acceso e itinerarios en la educación superior, destacando la trayectoria y facilidad que ofrece la educación a distancia permitiendo a los estudiantes obtener un doble diploma otorgado en dos países, así como el reconocimiento del aprendizaje y la experiencia adquiridos fuera de la educación tradicional.

Han de elaborarse curricula que sean más relevantes y basados en la demanda externa, diseñando programas que complementen la educación científica y tecnológica con aspectos socio-económicos, culturales y éticos que mejoren la calidad y eficacia en la relación con los demás actores socio-económicos.

No hemos de olvidarnos del estatuto docente. Se precisa que el acceso a la docencia atienda los criterios académicos de concurso público, oposición y/o antecedentes y titulación específica y que para la promoción docente se tomen en consideración: titulación con grado universitario, producción científica, formación de recursos humanos e innovación pedagógica.

El docente debe estar actualizado en las corrientes teóricas de su conocimiento, en nuestro caso las Ciencias Sociales tanto a nivel nacional como internacional y tener una postura abierta a la reflexión, la auto-reflexión y a la crítica, Manifestando su apertura al cambio, a la creatividad, a la solidaridad y a los valores democráticos, con respeto a la identidad cultural. Ha de tener experiencia en su área de conocimiento para lograr una formación efectiva en el futuro profesional y demostrar su capacidad de asimilación de los cambios para asegurar así, la transmisión de contenidos actualizados.

En lo que se refiere al particular método de enseñanza a distancia de nuestra universidad, deberíamos destacar la necesidad del dominio de las metodologías pertinentes al trabajo en equipo y la producción de textos propios académicos adecuados a este tipo de enseñanza, asumiendo una actitud activa y comprometida con estos principios. El profesor en este tipo de universidades de enseñanza a distancia precisa la perspectiva de trabajar como orientador del proceso de enseñanza-aprendizaje, reubicando el papel del profesor. De este modo, ha de conseguirse que, profesores y alumnos, queden 
envueltos en lo que podríamos denominar la creación/producción del conocimiento de manera conjunta, con el propósito principal de que la realidad sea entendida y aprendida y no solamente reproducida.

Las actividades de investigación deben integrarse en proyectos homologados y deberían ser necesariamente objeto de comunicación y divulgación científica, debiéndose, igualmente, considerar la inclusión de las actividades de investigación, extensión y perfeccionamiento de postgrado en la carga horaria de los profesores. Igualmente, ha de tomarse en consideración, la evaluación del desempeño docente, tanto de los iguales como de los alumnos así como de la evaluación externa y la auto evaluación.

Las Universidades, en definitiva, deben promover políticas y programas continuos de formación y capacitación docente tanto en la especialidad como en aspectos didáctico-pedagógicos para optimizar la labor docente tanto en los aspectos didácticos como en el uso de recursos tecnológicos y disposición de la infraestructura necesaria en cualquiera de las prácticas pedagógicas.

Por lo tanto, han de imponerse nuevas proposiciones estratégicas de la enseñanza. Así, ha de fomentarse en los alumnos el pensamiento crítico y la capacidad de reflexión tan escasa en muchos estudiantes universitarios. Promover ámbitos de discusión de las cuestiones político-sociales y económicas relacionadas con su medio y vivenciar los contenidos de los fundamentos teóricos a través de situaciones reales y actuales. Y ha de proporcionarse un apoyo académico efectivo a los alumnos e incentivar el trabajo en equipos interdisciplinarios de los mismos. Contextualizar la enseñanza a las particularidades de cada tema concreto de enseñanza e incentivar el desarrollo personal y colectivo de los alumnos. Igualmente se hace necesario la revalorizar el papel del docente universitario como creador de conocimiento e impulsor de ideas y análisis crítico

\section{BIBLIOGRAFIA}

Administración Nacional de Educación Pública. Consejo Directivo Central, «Comisión de Transformación de la Educación Media Superior, algunos elementos para la discusión acerca de la estructura curricular de la Educación Media Superior».

ANDY GREEN, «Education, Globalization and the Nation State» (Houndmills: Macmillan Press, 1997).

CARLOS A. CUllen, «Los contextos de la integración educativa. De la bipolaridad a la multipolaridad" Revista latinoamericana de innovaciones educativas (1995). 
«Educación y procesos de integración económica: el caso del Mercosur» La educación. Revista Interamericana de Desarrollo Educativo (1993).

GREG DIMITRIADIS y GEORGE KAMBERELIS, «Shifting terrains: Mapping education within a global landscape" Annals of the American Academy of Political and Social Science 551 (1997),

Groupe de Lisbonne (dir. Riccardo Petrella), «Limites à la Compétitivité Pour un nouveuan contrat mondial», Labor, Bruxelles, 1995, p. 60.

JORGE RAMIRO SABOGAL VARCE y otros. "Opiniones y sugerencias como aportes al proceso de reforma curricular en la Facultad de Derecho y Ciencias Políticas de la Universidad de Antioquia». Universidad de Antioquia. Facultad de Derecho y Ciencias Políticas. Medellin. Marzo 2005.

JOAN SPERO, "The challenge of globalisation» US Department of State Dispatch 7:40 (1996), pp. 481-484.

Manuel CaSTElls, "The Network Society", Volumen I de la obra The Information Age: Economy, Society and Culture, Blakwell, 1996.

M. Pereyra y otros (comp.), «Globalización y descentralización de los sistemas educativos» (Barcelona: Pomares-Corredor, 1996).

"Memoria anual de la Comisión Nacional de Educación y acreditación universitaria». Ministerio de educación, ciencia y tecnología».

Noam Chomsky y Heinz Dieterich, "La aldea global «(Tafalla: Txalaparta, 1997).

Peter A. Hall y Sidney Tarrow, «Globalisation and area studies: When is too broad too narrow" The Chronicle of Higher education (23 de enero de 1998).

PETER DRUCKER, «La sociedad poscapitalista» (Buenos Aires: Editorial Sudamericana, 1995.

ROBERT BOYER "Les mots et les Réalités" in Mondialization - Au-de lá des myths», La Découvert \& Syros, Paris, 1997. p. 29.

«The Inter-State Structure of the Modern World System» in International Theory, Cambridge University Press, 1996.

Wallerstein: "Le Système du Monde», Flammarion, París, 1980-1985.

«V Conferencia Iberoamericana de Educación. Declaración de Buenos Aires» en Revista Iberoamericana de Educación núm. 9 (Sept-Dic. 1995),

«VI Conferencia Iberoamericana de Educación. Declaración de Concepción» en Revista Iberoamericana de Educación núm. 12 (Sept-Dic. 1996).

"VII Conferencia Iberoamericana de Educación. Declaración de Mérida» en Revista Iberoamericana de Educación núm. 15 (Sept.-Dic. 1997).

"VII Conferencia Iberoamericana de Educación. (Sintra Portugal) Organización de Estados Iberoamericanos. 